\title{
Rheological interfacial properties of espresso coffee foaming fractions
}

\author{
Piazza, L., Bulbarello, A. and Gigli, J. \\ Department of Food Science and Microbiology (DISTAM), \\ University of Milan, via Celoria 2, 20133 Milan, Italy \\ Correspondent e-mail: laura.piazza@unimi.it; Fax: +39.02.50316632
}

\begin{abstract}
Coffee foaming fractions (total and its sub-fractions: proteins/melanoidins fraction and polysaccharides fraction) were extracted from defatted and raw commercial roasted ground coffee. All the extracted fractions were analyzed using an interfacial rheometer CIR 100 (FTA) operating in time sweep mode, in order to study the viscoelastic behaviour of the surface-adsorbed layer. The proteins/melanoidins component of the coffee foaming fraction exhibits the highest viscoelastic interfacial properties. The kinetic of the film formation is mediated by the polysaccharide component. Lipids have a mayor role in the interfacial film formation. Correlations were established between air/water interface properties of the foaming systems and the respective foam volume by means of the image analysis. It resulted that the protein/melanoidins fraction is mainly responsible for the coffee foamability.
\end{abstract}

Keywords: Interfacial Rheology, Espresso Coffee, Image Analysis, Foam

\section{Introduction}

The espresso coffee beverage is a complex dispersion where the liquid phase is topped by a thick layer of foam of tiny bubbles. Espresso coffee foam is more typically refereed as "cream".

It has been stated that a correlation exists between foamability and the products of the Maillard reaction that occurs during green coffee roasting, but no results have been published about the mechanism of foam formation and foam stabilization (Petracco et al., 1999). Furthermore, it is well known that lipids are present in espresso coffee brewing as a microemulsion (Illy and Viani, 1995), but their role on foam formation is not described.

The stability and instability of foams are at great extent determined by the surface rheological properties of adsorption layers at the liquid interface because, due to the number of bubbles or droplets in the system, the interfacial area can be enormous. The structure and properties of the interface affect many aspects of the physical properties of foam and emulsions (Collyer and Clegg, 1998). Melanodins, proteins, polysaccharides and lipids diffuse from the liquid phase of espresso to the liquid/air surface and develop a thin film that can be investigated by means of advanced physical techniques, as the dynamic oscillatory spectrometry of the interface (Burgess and Ozlen, 1997). Interfacial rheology is the study of the deformation and flow of 
infinitesimally thin films of material at liquid/gas or liquid/liquid interface. It has been applied to get information about the kinetics of the surface film formation in espresso coffee.

\section{Materials and Methods}

\section{Extraction and isolation of roasted coffee foaming fractions}

Foaming fractions with or without lipids were extracted from ground dark roasted coffee blend (70\% Coffea Robusta and 30\% Coffea Arabica):

1) to get the defatted foaming fraction according to Petracco (Petracco et al., 1999) in order to obtain: the Total Foaming Fraction (TFF), the Foaming Fraction A (FFA) with a higher carbohydrate-like character and the Foaming Fraction B (FFB) with a higher melanoidin-like character;

2) to get the foaming fraction in presence of lipids the previous procedure was followed except for the Soxhlet fat extraction step.

\section{Interfacial rheology measurements}

Aqueous solutions $(0.5 \% \mathrm{w} / \mathrm{w})$ were prepared for all the foaming fraction previously described and were analyzed by means of the CIR-100 interfacial shear rheometer (FTA, Portsmouth Virginia, USA) fitted with a Pt/Ir Du Noüy ring geometry. Bi-dimensional dynamic moduli $G_{s}$ ' and $G_{s}{ }^{\prime \prime}(\mu N / m)$ were recorded $v s$ time (s) during time sweep test up to $18000 \mathrm{~s}$ at an oscillation frequency of $3 \mathrm{~Hz}$ and an amplitude of $5000 \mu \mathrm{rad}$. Each data point was averaged over three cycles. An air/water interface was used as a reference for all experiments. All time sweep tests were performed at $20 \pm 0.2^{\circ} \mathrm{C}$.

\section{Image analysis measurement}

Foaming fraction solutions prepared for the rheological tests were stirred in a Plexiglas vessel of rectangular section in standard conditions to get foams. Images of the foams were acquired by means of a scanner (19200 trust, USA) at different times ranging from 40 to $1860 \mathrm{~s}$ after foam formation.

Images were processed using Image Pro Plus ver. 5.0 (Media Cybernetics, Inc) and frontal foam area was expressed in pixel.

\section{Results and Discussion}

The dense, reddish-brown foam that tops the bulk liquid phase of espresso coffee is composed mainly of tiny carbon dioxide from the Maillard's reaction that takes place during the roasting of green coffee, and water vapour bubbles arranged in a honeycomb structure. Bubbles are surrounded by surfactant films in a dispersed phase formed by small oil droplets in emulsion. They are perceived in the mouth as a special creamy sensation and includes emulsified oils containing aromatic compounds (Illy and Viani, $1995)$. 


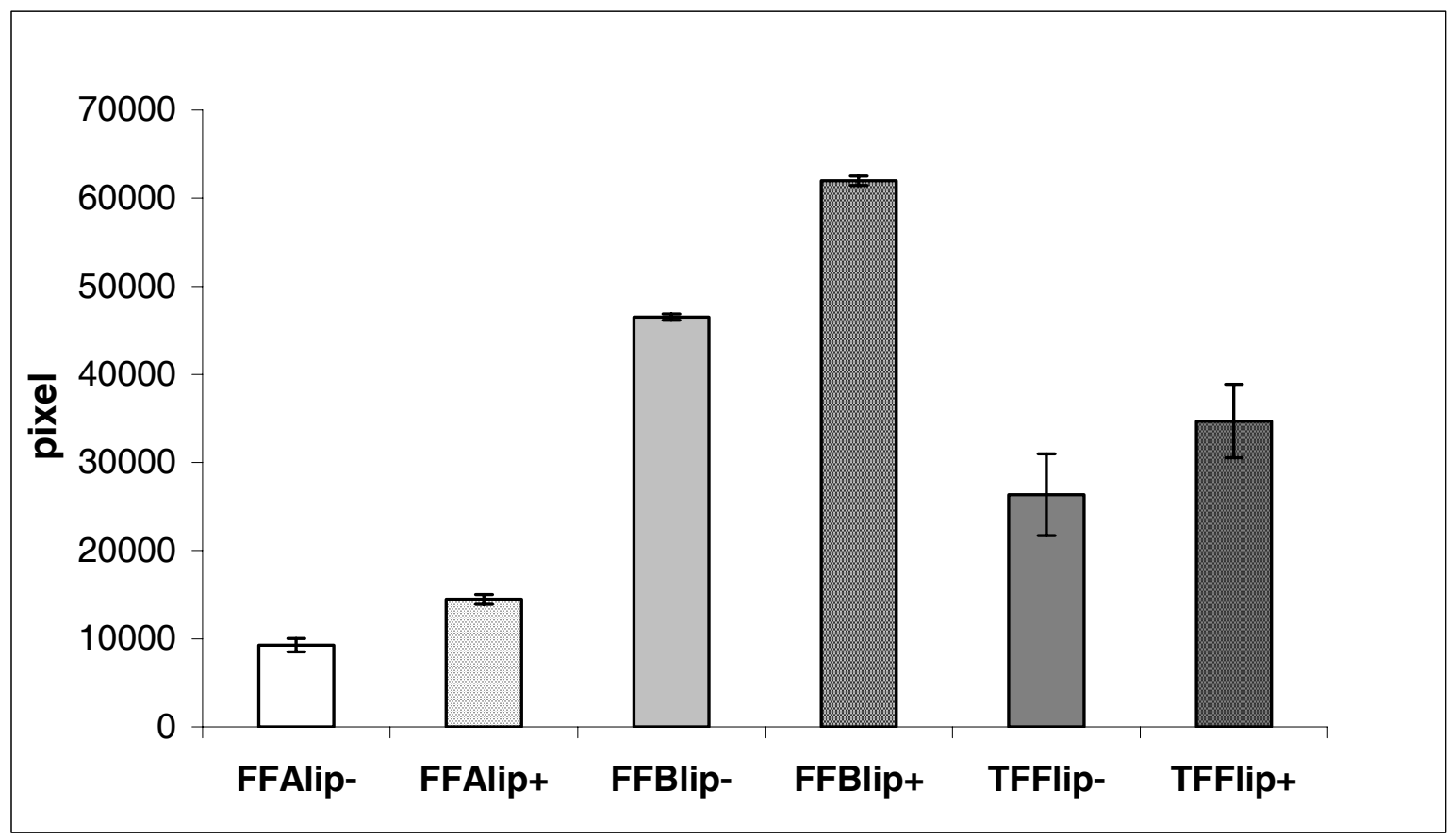

Figure 1. Area of foams (see text) for different foaming fractions with (lip+) or without (lip-) lipids.

In figure 1 the foamability (ease and extent of foam formation) of different espresso foaming fractions extracted from roasted ground coffee are summarized, both in presence or absence of lipids. Melanoidins rich solutions (FFB fractions) show the highest volumes of foam generated by a standard stirring procedure.Due to a combination of pronounced surface activity and ability to form thick viscoelastic layers, most soluble food proteins adsorb rapidly at the air-water interface during aqueous foam formation, leading to steric stablization of the resulting bubbles against coalescence and disproportionation. FFA fractions with or without lipids, whose carbohydratelike characters has been described, express the worst performance, while an intermediate behaviour can be observed for the total foaming fraction TFF.

The chemical nature of surface-active agents in the espresso coffee brew has not been satisfactorily clarified. The roasting process modify proteins by pyrolitic reactions and induce polysaccharides depolymerization, structural modifications and formation of condensation complexes with proteins along with the browning reactions giving melanoidins.

Data presented in figure 1 indicate that the foamability is more consistent if lipids are not separated from the FFB fraction. This is true for the TFF and FFA fractions, too.

Triglycerides (with a small amount of free fatty acids, phosphatides, mono and diglycerides) and diterpens alcohol esters are the major lipid classes in coffee brewed from ground coffee beans (Ratnayake, et al. 1993). It might be hypothesized a carrier role of surface active compounds towards the air/water interface charged to microemulsified oil droplets. 
The results of dynamic interfacial experiments are illustrated in figures $\mathbf{2}$ and 3 which show the surface storage modulus $G_{s}$ ' of foaming fraction plotted vs the time.

In the case of the foaming fraction with melanoidins as active component, the formation of a gel-like layer at the interface is indicated by the rapid increase in the interfacial elastic modulus $G_{s}$ '. The value of $G_{s}$ ' exceeds the upper limit of the instrument $(20 \mathrm{~m} \mathrm{~N} / \mathrm{m})$ within just $11000 \mathrm{~s}$. In the absence of proteins, the foaming fraction FFA, rich in polysaccharides, does no give rise at molecular interaction at the interface with elastic properties.

The kinetics of surface film formation is lowered in the Total Foaming Fraction (TFF) and after $18000 \mathrm{~s}$ the absolute value of $\mathrm{G}_{\mathrm{s}}$ ' is still low. Moreover we can observe a "lag phase" in the viscoelastic properties development. It is presumably due to thickening role of polysaccharides that results in an increase in the bulk viscosity and hence in a lower diffusion of surface active compounds to the air/water interface. At very short time the adsorption process is strongly influenced by the availability in solution of such molecules. This aspect is at present under investigation.

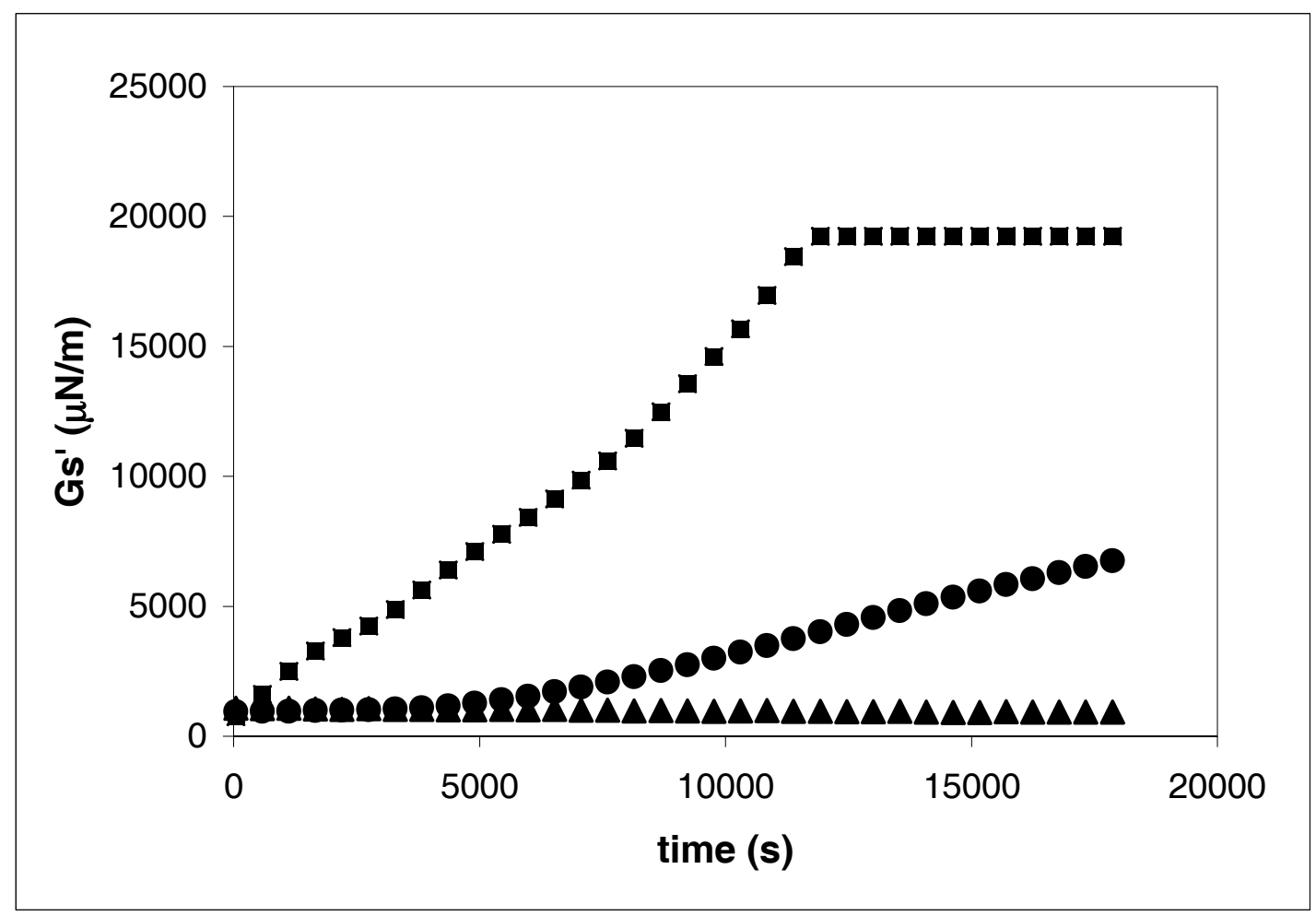

Figure 2. Interfacial dynamic elastic modulus $\left(\mathrm{G}_{\mathrm{s}}{ }^{\prime}\right)$ of foaming fractions with lipids: $\square$ FFBlip+; $\bullet$ TFFlip+; $\triangle$ TFFlip+.

A trend of the interface elastic modulus, which is similar in the shape but higher in intensity with respect to the previously discussed one, is finally shown in figure 3 for the FFB fraction lipids free in comparison to the raw FFB solution. The surface properties of surfactants can be explained by important molecular mechanism such as conformational changes of high 
molecular weight substances like proteins in presence of lipids (Rodriguez and Rodriguez, 1995) that affect surface film structure and film formation capacity.

It is clear that the stability of a foam containing emulsified fat particles may depend on both the size and the composition of the dispersed lipid particles.

In conclusion, the macroscopic evaluations of foamability of all the studied foaming systems that were presented as the result of the image analysis of the foams are widely confirmed.

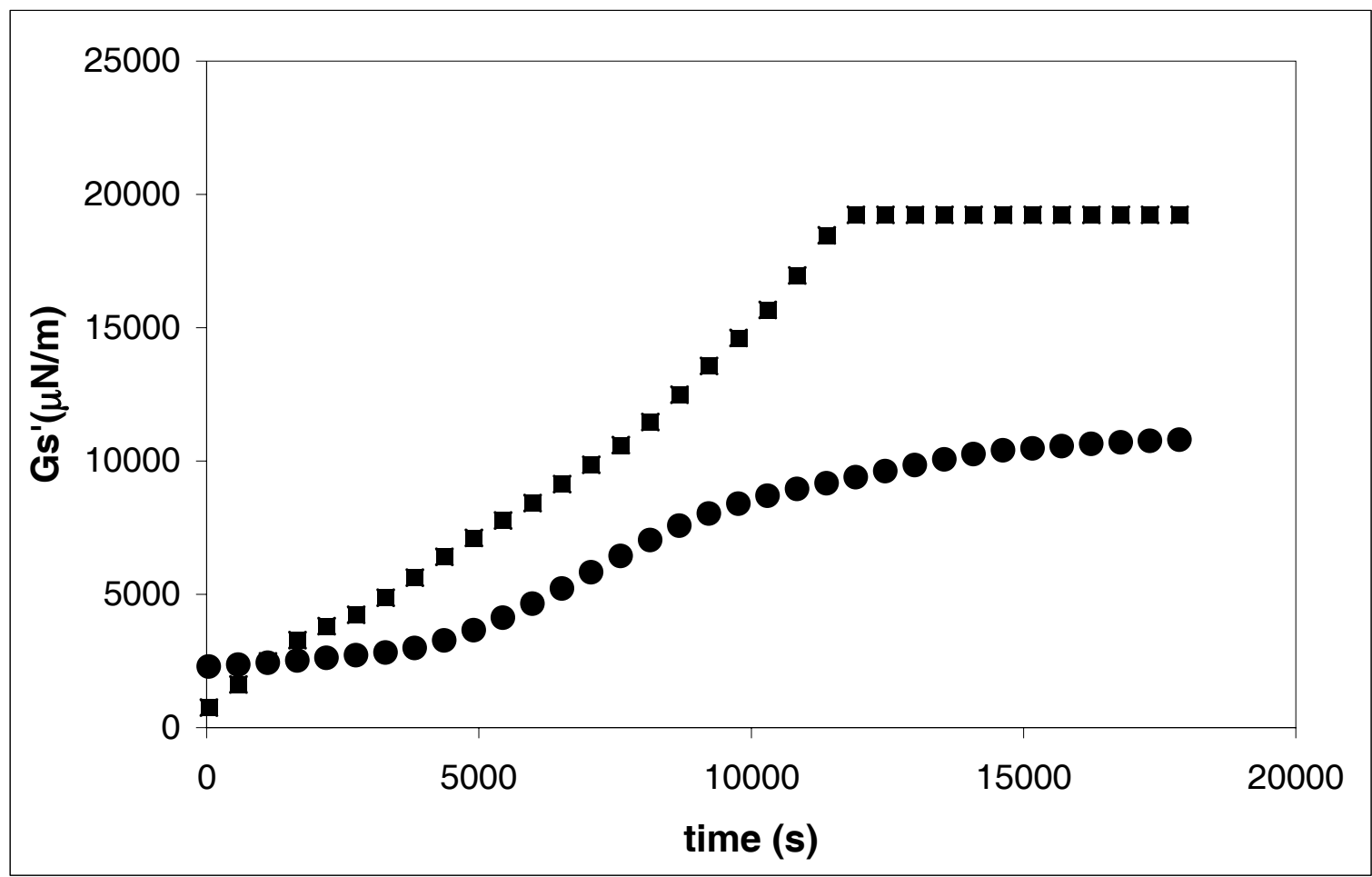

Figure 3. Interfacial dynamic elastic modulus $\left(\mathrm{G}_{\mathrm{s}}{ }^{\prime}\right)$ of foaming fraction (FFB) with and without lipids: EFFBlip+; • FFBlip-.

\section{Conclusions}

Our surface rheology data can provide, at the moment, only phenomenological observations of changing interactions between neighbouring polymer molecules in protein/polysaccharides/lipids foaming systems that were isolated from the roasted ground coffee.

An important result of this study is that the relative strength of the twodimensional physical network at the air/water interface is dependent on the interaction between polymers and lipids. 


\section{References}

Burgess, D.J. and Ozlen S.N. (1997). Interfacial Rheological and Tension Properties of Protein Film. Journal of Colloids and Interface Science, 189:74.

Collyer, A.A. and Clegg, D.W. (1998). Rheological measurement. Chapman \& hall, London, p.723.

Illy, A. and Viani, R. (1995). Espresso Coffee: The Chemistry of Quality. Academic Press, London, p.294.

Petracco, M., Navarini, L., Abatangelo, A., Gombac, V., D’Agnolo, E. and Zanetti F. (1999). Isolation and characterization of a foaming fraction from hot water extracts of roasted coffee. Colloq. Sci. Int. Cafe $18^{\text {th }}: 95$.

Ratnayake, W.M., Hollywood, R., O’Grady, E. and Stavric, B. (1993). Lipid content and composition of coffee brews prepared by different methods. Food Chem. Toxicol, 31:263.

Rodriguez, P.J.M. and Rodriguez, N.M.A. (1995). Protein adsorption and protein-lipid interaction at the air-aqueous solution interface. Colloids and interfaces A, 103: 91. 